\title{
Geometric Hall effects in topological insulator heterostructures
}

\author{
K. Yasuda ${ }^{1 \star}$, R. Wakatsuki ${ }^{1}$, T. Morimoto ${ }^{2}$, R. Yoshimi ${ }^{1}$, A. Tsukazaki ${ }^{3,4}$, K. S. Takahashi ${ }^{2}$, M. Ezawa ${ }^{1}$, \\ M. Kawasaki ${ }^{1,2}$, N. Nagaosa ${ }^{1,2}$ and Y. Tokura ${ }^{1,2}$
}

Geometry, both in momentum and in real space, plays an important role in the electronic dynamics of condensed matter systems. Among them, the Berry phase associated with nontrivial geometry can be an origin of the transverse motion of electrons, giving rise to various geometric effects such as the anomalous', spin $^{2}$ and topological Hall effects $^{3-6}$. Here, we report two unconventional manifestations of Hall physics: a sign-reversal of the anomalous Hall effect, and the emergence of a topological Hall effect in magnetic/non-magnetic topological insulator heterostructures, $\mathrm{Cr}_{x}\left(\mathrm{Bi}_{1-y} \mathrm{Sb}_{y}\right)_{2-x} \mathrm{Te}_{3} /\left(\mathrm{Bi}_{1-y} \mathrm{Sb}_{y}\right)_{2} \mathrm{Te}_{3}$. The sign-reversal in the anomalous Hall effect is driven by a Rashba splitting at the bulk bands, which is caused by the broken spatial inversion symmetry. Instead, the topological Hall effect arises in a wide temperature range below the Curie temperature, in a region where the magnetic-field dependence of the Hall resistance largely deviates from the magnetization. Its origin is assigned to the formation of a Néel-type skyrmion induced by the Dzyaloshinskii-Moriya interaction.

The geometry and topology in Hilbert space constitute a central issue in quantum physics, which has recently also shed a new light on the electronic states in solids. The wavefunctions are characterized by the Berry connection between two neighbouring points, in both momentum space and real space, which plays the role of the vector potential leading to the concept of emergent electromagnetic field (EEMF). Global topology of the manifold in Hilbert space is represented by topological integers. As integers cannot change continuously, it gives a certain stability to the system. For example, the Chern number, which is given by the integral of the emergent magnetic field over the first Brillouin zone, protects the surface or edge states supporting the dissipationless current flows (that is, bulk-edge correspondence). The one-dimensional chiral edge mode in the quantum Hall effect and the Dirac surface state in three-dimensional topological insulators (TI; refs 7,8) are the representative examples of this phenomenon. In addition, it has recently been proposed ${ }^{9,10}$ and observed ${ }^{11-14}$ that TI with doped magnetic ions-namely, $\mathrm{Cr}$ or $\mathrm{V}$ - produces the quantized version of the anomalous Hall effect (AHE) in the absence of an external magnetic field.

Topology in real space, on the other hand, is exemplified by skyrmion spin texture ${ }^{15-17}$ found in chiral-lattice magnets such as $\mathrm{MnSi}$ (ref. 15) or $\mathrm{Fe}_{1-x} \mathrm{Co}_{x} \mathrm{Si}$ (ref. 16). Here, the solid angle subtended by the spins forms an emergent magnetic field in real space, and its integral over the two-dimensional space defines a topological integer called the skyrmion number. Namely, the skyrmion number counts the number of times the spin direction wraps around a unit sphere. This integer protects the skyrmion from annihilation and allows it to behave as a single particle.

Thus far, the EEMF in momentum and real spaces have mostly been studied separately. Recent advances in fabricating artificial structures of materials, however, enable the design of emergent phenomena considering both momentum and real spaces in a unified way. Here, we examined these geometric Hall effects in TI heterostructures composed of magnetic TI $\mathrm{Cr}_{x}\left(\mathrm{Bi}_{1-y} \mathrm{Sb}_{y}\right)_{2-x} \mathrm{Te}_{3}$ (CBST) and non-magnetic $\mathrm{TI}\left(\mathrm{Bi}_{1-y} \mathrm{Sb}_{y}\right)_{2} \mathrm{Te}_{3}$ (BST; refs 11-13,18-22), which were grown on $\operatorname{InP}(111)$ substrates using molecular-beam epi$\operatorname{taxy}^{20-22}$. By applying a field-effect transistor structure (FET, see Methods), the Fermi energy $\left(E_{\mathrm{F}}\right)$ of the TI heterostructures can be precisely controlled in a large energy range over the bulk bandgap. In particular, the quantum AHE realized in the magnetic TIs offers an interesting scenario where the two different physical mechanisms merge-namely, skyrmion formation around the doped carrier into the quantum Hall ferromagnet ${ }^{23}$ and skyrmion formation at the interface of magnets ${ }^{24-27}$. First, we investigate the gate voltage $\left(V_{\mathrm{G}}\right)$ and temperature $(T)$ dependence of the Hall effect for a 2-nm CBST/5-nm BST heterostructure as shown in Fig. 1a. With increasing $V_{\mathrm{G}}$ at $2 \mathrm{~K}$ (Fig. 1b), the hysteresis curves of the Hall conductivity $\sigma_{x y}$ gradually change shape, demonstrating a maximum value at $V_{\mathrm{G}}=0.2 \mathrm{~V}$. In Fig. $1 \mathrm{c}, \mathrm{d}$, we plot the temperature dependence at selected voltages $V_{\mathrm{G}}=0.2 \mathrm{~V}$ and $-7.0 \mathrm{~V}$. In contrast to the conventional temperature dependence of magnetic TI shown in Fig. 1c at $V_{\mathrm{G}}=0.2 \mathrm{~V}$, where $E_{\mathrm{F}}$ is close to the Dirac point, we find two notable features under hole accumulation at $V_{\mathrm{G}}=-7.0 \mathrm{~V}$ in Fig. $1 \mathrm{~d}$ : first, sign-reversal of the anomalous Hall conductivity $\sigma_{x y}^{\mathrm{A}}$ (Hall conductivity under zero magnetic field) at $6 \mathrm{~K}$ and, second, nonmonotonous behaviour of $\sigma_{x y}$ as a function of magnetic field. In this paper, we first study the former feature and discuss the latter afterwards, both from the viewpoint of the geometric Hall effect. The detailed data set of $\sigma_{x y}^{\mathrm{A}}$ in $T-V_{\mathrm{G}}$ parameter space (Fig. 1e) shows the sign-reversal roughly at negative $V_{\mathrm{G}}$ and in the $6-20 \mathrm{~K}$ region. Such a sign-reversal of $\sigma^{\mathrm{A}}{ }_{x y}$ has not been observed in singlelayer CBST, which always gives a positive sign regardless of its $E_{\mathrm{F}}$ position and temperature ${ }^{11-13}$, as exemplified in Fig. 2a. Hence, such a transition between positive and negative $\sigma_{x y}^{\mathrm{A}}$ is a distinct behaviour in the 2-nm/5-nm (Fig. 1d) and 3-nm/5-nm (Fig. 2b) heterostructures. Note that the longitudinal conductivity (approximately $\left.10^{2}(\Omega \mathrm{cm})^{-1}\right)$ is so low that each anomalous component cannot be of an extrinsic origin ${ }^{1}$, such as skew scattering; therefore, an intrinsic origin should be explored in the electronic band structure characteristic of the TI heterostructures.

\footnotetext{
${ }^{1}$ Department of Applied Physics and Quantum-Phase Electronics Center (QPEC), University of Tokyo, Tokyo 113-8656, Japan. ${ }^{2}$ RIKEN Center for Emergent Matter Science (CEMS), Wako 351-0198, Japan. ${ }^{3}$ Institute for Materials Research, Tohoku University, Sendai 980-8577, Japan. ${ }^{4}$ PRESTO, Japan Science and Technology Agency (JST), Chiyoda-ku, Tokyo 102-0075, Japan. ${ }^{\star}$ e-mail: yasuda@cmr.t.u-tokyo.ac.jp
} 


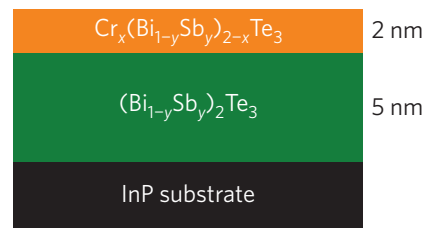

e

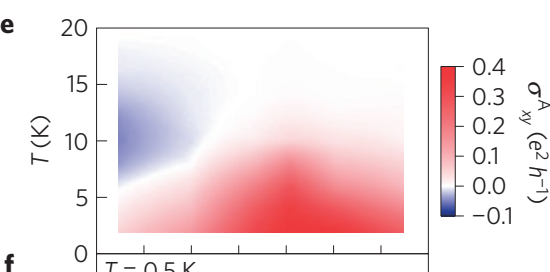

f

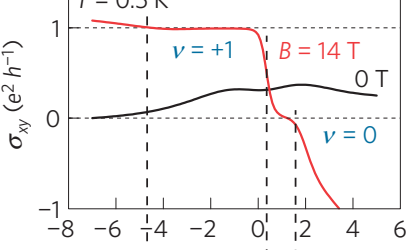

g

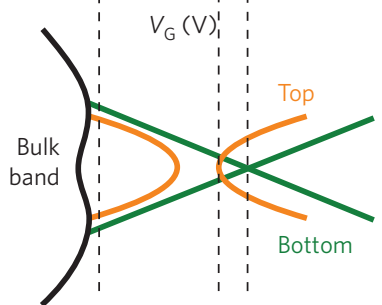

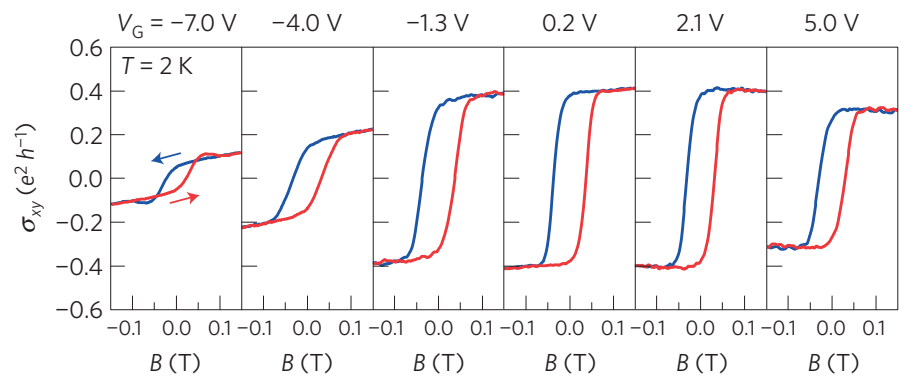

c

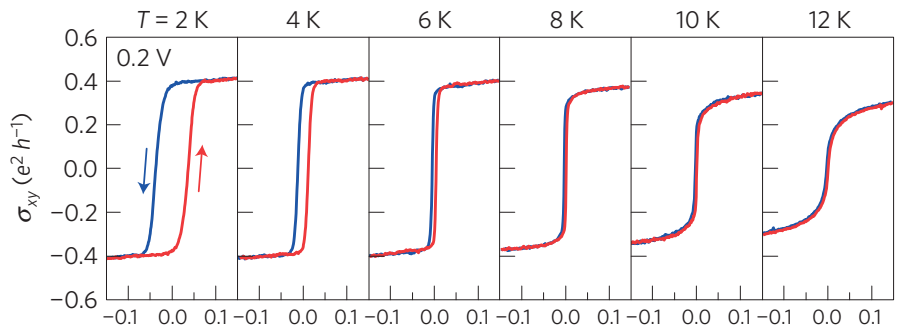

d

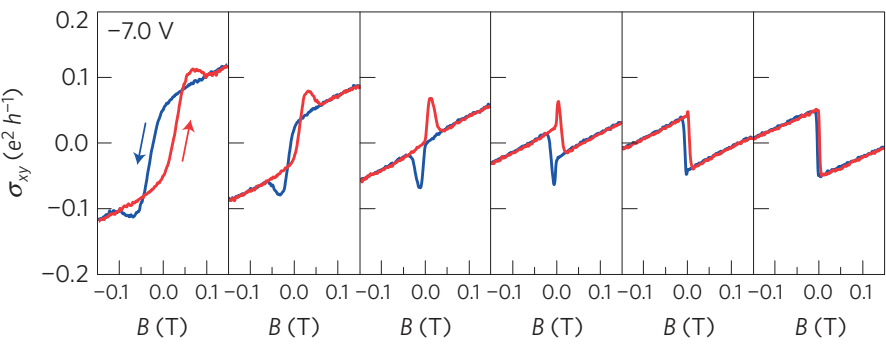

Figure 1 | Anomalous Hall conductivity of a topological insulator (TI) heterostructure. a, A schematic picture of a TI heterostructure. b, Magnetic-field dependence of the Hall conductivity $\sigma_{x y}$ at various gate voltages $V_{G}$ at $T=2 \mathrm{~K}$. A red (blue) curve represents the process of increasing (decreasing) magnetic field. $\mathbf{c}, \mathbf{d}$, Magnetic-field dependence of $\sigma_{x y}$ at various temperatures for $V_{G}=0.2 \mathrm{~V}(\mathbf{c})$ and $V_{G}=-7.0 \mathrm{~V}$ (d). e, Colour contour plot of the anomalous Hall conductivity $\sigma^{A} x y$ as a function of $V_{G}$ and temperature. Red (blue) regions corresponds to positive (negative) $\sigma^{A}{ }_{x y}$. $\mathbf{f}$, Hall conductivity as a function of $V_{\mathrm{G}}$ at $T=0.5 \mathrm{~K}$ at fields of $B=0 \mathrm{~T}$ (black) and $B=14 \mathrm{~T}$ (red), showing $v=+1$ and $v=0$ quantum Hall plateaux, from ref. 20. $\mathbf{g}$, Assumed schematic band structure of the heterostructure, showing the bulk band (black), the top surface state (brown) and the bottom surface state (dark green).

To address this issue, we theoretically study the low-energy effective Hamiltonian of the TI heterostructures (see Methods). We consider two cases, a 10-nm CBST single layer and a 5-nm CBST/5-nm BST heterostructure, as shown in Fig. 3a,d. The band structure obtained for CBST (Fig. 3b) shows gapped Dirac surface bands in addition to the bulk bands. In the case of CBST/BST (Fig. 3e), the Dirac band at the top surface of the CBST is gapped owing to the exchange coupling, whereas the Dirac band at the bottom surface of the BST remains gapless. Moreover, the top of the valence bands shows Rashba splitting because of the broken spatial inversion symmetry in the heterostructures (red lines in the lower inset of Fig. 3e), and these Rashba split bands are slightly gapped owing to the exchange coupling (black lines). In fact, such Rashba splitting in the bulk band of TI has also been observed in previous angle-resolved photoemission spectroscopy (ARPES) studies ${ }^{28}$. In Fig. $3 c$,f, we show the Hall conductivity obtained from the Berry curvature that encodes the nontrivial geometry in the momentum space. In both cases, $\sigma_{x y}$ is maximized when $E_{\mathrm{F}}$ is within the gap of the surface bands. In addition, $\sigma_{x y}$ in Fig. $3 \mathrm{f}$ shows a sharp negative peak at the top of the valence bands, denoted by a red triangle. This is attributed to the slightly gapped Rashba split bands on which the Berry curvature is concentrated. Moreover, the negative peak decreases for larger exchange coupling, which corresponds to the case of lower temperature (see Supplementary Information for the details). This means that a positive AHE component is dominant at lower temperatures. These theoretical arguments coincide well with the experimental results shown in Fig. 1e,g, in which the band energy scheme is based on the results of $\sigma_{x y}$ at zero and high
(14 T) fields, including $v=1$ and $v=0$ quantum Hall plateaux (Fig. 1f; ref. 20).

In addition to the sign-reversal, we observe anomalous magneticfield-dependent behaviours in the Hall effect in 2-nm CBST/5-nm BST heterostructures, as shown in Figs $2 \mathrm{c}$ and $1 \mathrm{~d}$. To elucidate the hysteretic anomaly in the $R_{y x}$ versus $B$ curve we compared $R_{y x}{ }^{\prime}$ $\left(=R_{y x}-R_{0} B\right)$ with the magnetization of the film (see Methods) in the top panel of Fig. 2d. Here, $R_{0}$ is the ordinary Hall coefficient determined from the linear slope at high magnetic field (see Supplementary Information). In the conventional theory, $R_{y x}{ }^{\prime}$ is nothing other than the anomalous Hall term, which is proportional to the magnetization $M$. In reality, however, a large discrepancy between $R_{y x}{ }^{\prime}$ and $M$ is discerned; we need to add the nontrivial Hall component (shown in the bottom panel of Fig. 2d) as well as the $M$-linear anomalous Hall term to reproduce the observed $R_{y x}{ }^{\prime}$. As a plausible origin, we propose skyrmion formation from both theoretical and experimental aspects. Under skyrmion spin texture, the moving electrons experience the EEMF, giving rise to an additional Hall component termed the topological Hall effect (THE, note that 'topological' is defined in real space $)^{3,4}$, which is observed in the skyrmion phase of some chiral-lattice magnets ${ }^{5,6}$ as well as in frustrated magnets endowed with scalar spin chirality ${ }^{29,30}$. The magnetic-field dependence of THE shows a hysteresis behaviour; the magnetic field showing the maximal THE is observed to shift slightly to lower field as a whole by up to $\sim 0.02 \mathrm{~T}$ with decreasing sweep rate from $2 \times 10^{-3} \mathrm{~T} \mathrm{~s}^{-1}$ to $3 \times 10^{-5} \mathrm{~T} \mathrm{~s}^{-1}$ (see Supplementary Information for the details). 
a

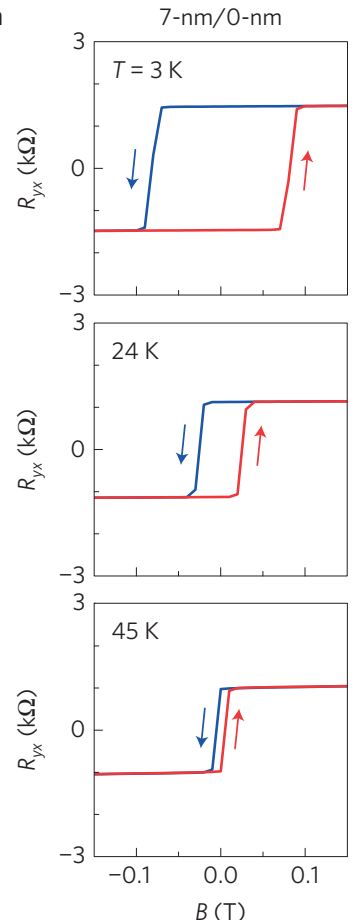

b
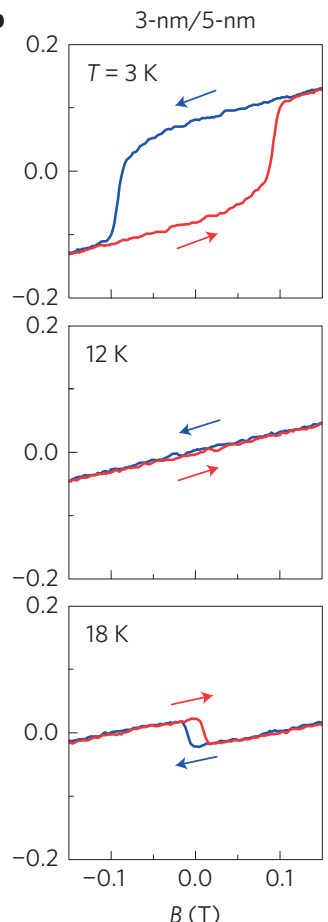
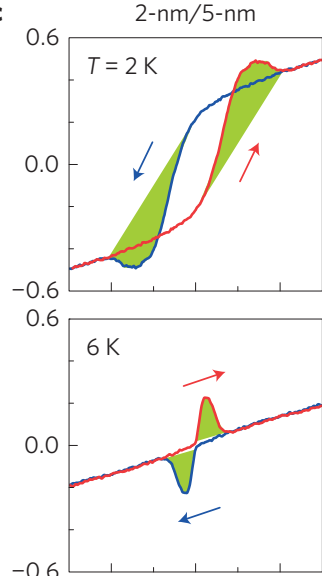$$
0.6
$$

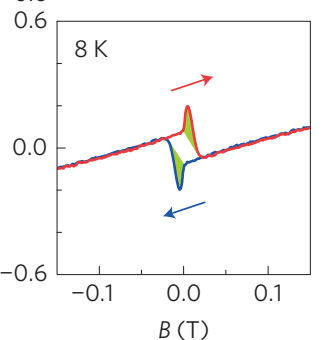

d
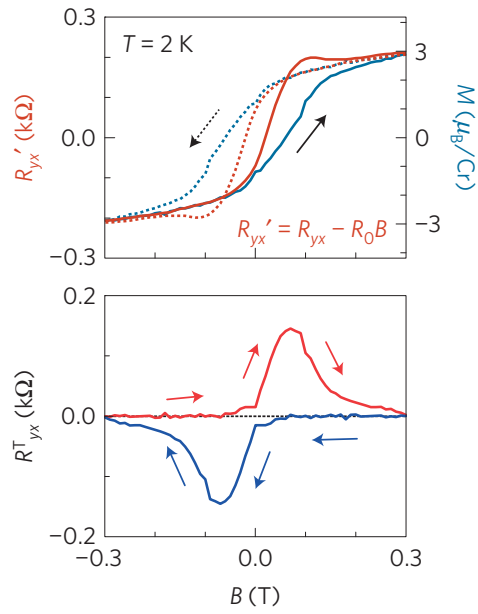

e

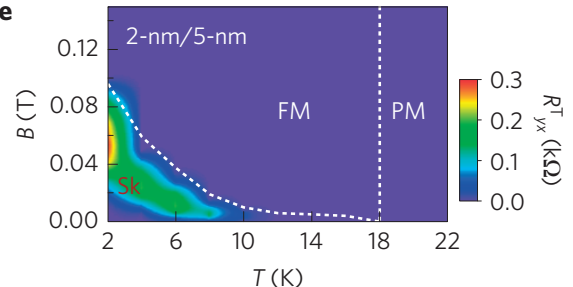

Figure 2 | Topological Hall effects of TI heterostructures. a-c, Magnetic-field dependence of the Hall resistance at various temperatures for single-layer CBST of thickness $7 \mathrm{~nm}$ (a), a 3-nm CBST/5-nm BST heterostructure (b) and a 2-nm CBST/5-nm BST heterostructure (c). d, (Upper panel) Magnetic-field dependence of $R_{y x}{ }^{\prime}$ (Hall resistance minus the normal Hall resistance, brown line) compared with the magnetization $M$ (blue line) for a 2-nm CBST/5-nm BST heterostructure. (Lower panel) Topological Hall component $R_{y x}^{\top}$. Red (blue) curve represents the process for increasing (decreasing) magnetic field. e, Magnetic-field and temperature dependence of $R_{y x}^{\top}$ highlighted in light green in c. Sk, FM and PM stand for skyrmion, ferromagnetic and paramagnetic, respectively. FM and PM states were determined from the temperature dependence of the Hall effect.

a

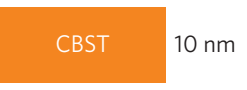

b
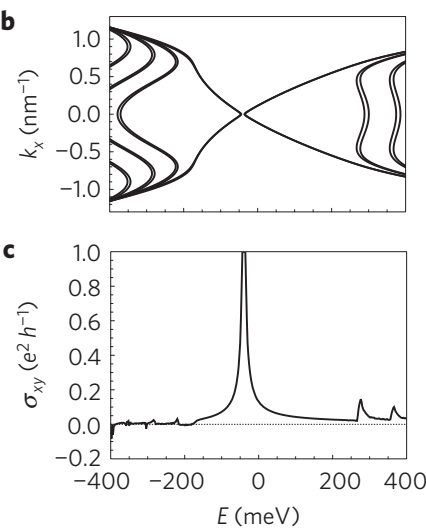

d
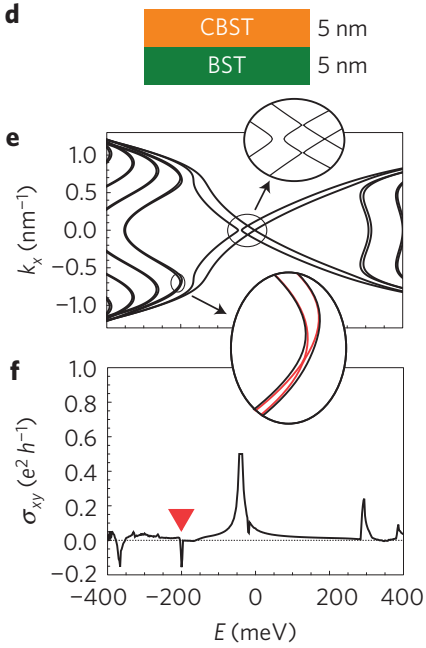

Figure 3 | Calculation of Hall conductivity. a,d, Schematic pictures of a CBST single layer with 10-nm thickness (a) and a CBST/BST heterostructure with 5-nm thickness in each layer (d). b,e, Calculated band structures for the single-layer CBST (b) and the CBST/BST heterostructure (e), where $k_{x}$ represents the in-plane momentum. The insets in e show enlarged images of the Dirac point (upper) and the top of the valence bands (lower) exhibiting Rashba splitting due to the inversion symmetry breaking. The energy bands at the top of valence bands without exchange coupling are simultaneously shown as red lines. c,f, Calculated anomalous Hall conductivity as a function of the Fermi energy $E$ for the single-layer CBST (c) and the CBST/BST heterostructure (f). The red triangle indicates the position of a negative contribution to the anomalous Hall conductivity.
To confirm the possibility of skyrmion formation, we simulated the energetic stability of a skyrmion using a three-dimensional tightbinding model (see Methods). We consider the following three cases: Bloch (Fig. 4a), Néel1 (Fig. 4b) and Néel2 (Fig. 4c) for a CBST single layer (Fig. 4d) and a CBST/BST heterostructure (Fig. 4h). In Fig. $4 \mathrm{e}-\mathrm{g}, \mathrm{i}-\mathrm{k}$, we show the formation energy of a skyrmion relative to that of the spin-collinear ferromagnet as a function of the skyrmion radius $R$ at various doping levels. For the CBST single layer in Fig. $4 \mathrm{e}-\mathrm{g}$, the energy is minimized always at $R=0$, implying that the ferromagnetic state is the ground state regardless of $E_{\mathrm{F}}$. For the electron-doped (Fig. 4i) or half-filling (Fig. 4j) cases of the CBST/BST heterostructure, the situation is similar. In the holedoped case in the heterostructure (Fig. $4 \mathrm{k}$ ), by contrast, a negative energy region exists for the Néel2-type skyrmion $(R \neq 0)$, denoted by a red triangle. The emergence of a stable condition for skyrmion formation can be explained as follows. The surface state of the TI exhibits inversion symmetry breaking and a fairly strong spin-orbit interaction, as exemplified by the spin-momentum-locking in the Dirac dispersion. Thus, the electrons at the surface state mediate the Dzyaloshinskii-Moriya (DM) interaction where the DM vector is pointing in the in-plane direction ${ }^{24-27,31}$. In the heterostructure, therefore, such an in-plane DM vector originating from the top CBST surface favours the formation of a Néel-type skyrmion ${ }^{24-27}$. However, in the single-layer CBST, DM vectors exist both at the top and bottom surface, each pointing along opposite directions, so that skyrmion formation is disfavoured owing to the frustration. Hence, broken spatial inversion symmetry associated with the heterostructure is the essential requirement for skyrmion formation.

Theoretical verification of skyrmion formation at the holeaccumulated condition agrees well with the experimental situation at large negative $V_{\mathrm{G}}(-7.0 \mathrm{~V})$, as shown in Fig. $1 \mathrm{~d}$. In 2-nm/5-nm heterostructures (Fig. 2c), the topological Hall components $R^{\mathrm{T}}{ }_{y x}$ are 


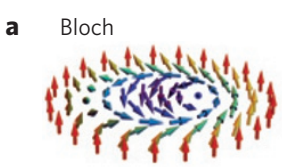

d

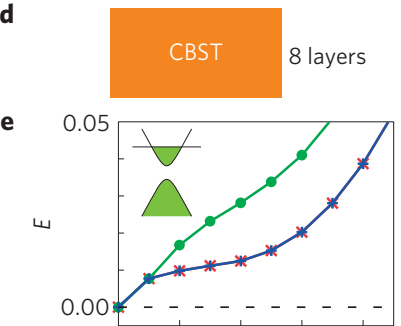

f
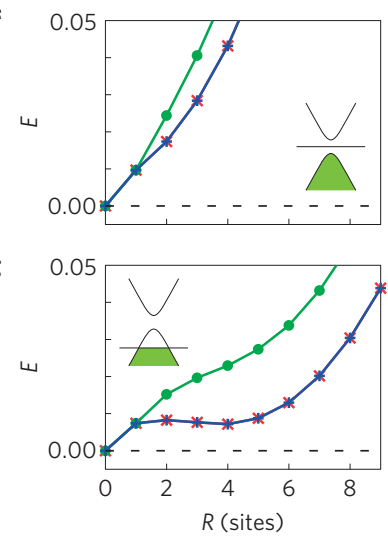

b Néel1

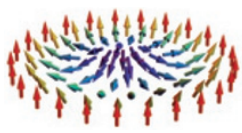

h
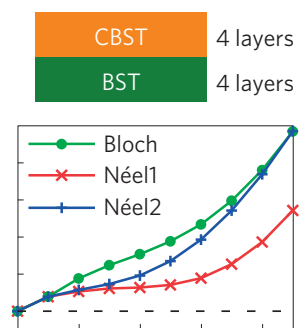

j

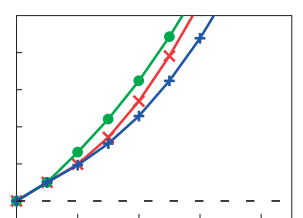

k

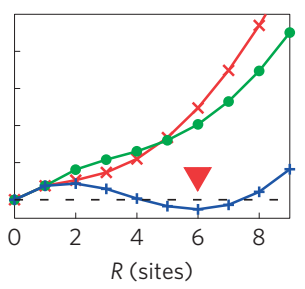

Figure 4 | Stability of a skyrmion on magnetic Tls. a-c, Schematic pictures of Bloch-type (a), Néel1-type (b) and Néel2-type (c) skyrmions. d, Schematic picture of a CBST single layer with eight sublayers. $\mathbf{e}-\mathbf{g}$, Calculated energy of a single skyrmion relative to that of the ferromagnet for the electron-doped case (e), the half-filling case (f) and the hole-doped case $(\mathbf{g})$ for the single-layer structure shown in $\mathbf{d}$ as a function of skyrmion radius $R$. $\mathbf{h}$, Schematic picture of a CBST/BST heterostructure with four sublayers of each. $\mathbf{i}-\mathbf{k}$, Same as $\mathbf{e}-\mathbf{g}$ for the electron-doped case (i), the half-filling case ( $\mathbf{j})$ and the hole-doped case ( $\mathbf{k})$ for the heterostructure shown in $\mathbf{h}$ as a function of skyrmion radius $R$. The red triangle shows the optimized $R$ to stabilize skyrmion formation. Green, red and blue lines in $\mathbf{e}-\mathbf{g}$ and $\mathbf{i}-\mathbf{k}$ correspond to Bloch-type, Néel1-type and Néel2-type skyrmions, respectively. $R$ is measured in units of the lattice spacing.

identified as the green region, which is shown in Fig. 2e in the $B-T$ parameter space. From this plot, we can see that skyrmions are formed in the course of magnetization reversal in a wide temperature range below the Curie temperature. The maximum THE, amounting to $140 \Omega$ (lower panel of Fig. 2 d), corresponds to the magnitude of an emergent magnetic flux density of $0.29 \mathrm{~T}$, taking into consideration the normal Hall coefficient of $490 \Omega \mathrm{T}^{-1}$ (see Supplementary Information). However, in the 3-nm CBST/5-nm BST heterostructure, no THE is observed, although sign-reversal of AHE is discerned, as shown in Fig. 2b. (As for the reproducibility of such a critical CBST-thickness-dependent THE, see Supplementary Information.) This observation implies the importance of the DM interaction; as the CBST thickness increases, the relative strength of the exchange interaction to the DM interaction increases, such that skyrmion formation would become less favoured. From this viewpoint, further optimization of the magnetic/non-magnetic TI heterostructure may open a way to control skyrmions on TI.

\section{Methods}

Methods and any associated references are available in the online version of the paper.
Received 25 August 2015; accepted 19 January 2016; published online 22 February 2016

\section{References}

1. Nagaosa, N., Sinova, J., Onoda, S., MacDonald, A. H. \& Ong, N. P. Anomalous Hall effect. Rev. Mod. Phys. 82, 1539-1592 (2010).

2. Jungwirth, T., Wunderlich, J. \& Olejník, K. Spin Hall effect devices. Nature Mater. 11, 382-390 (2012).

3. Bruno, P., Dugaev, V. K. \& Taillefumier, M. Topological Hall effect and Berry phase in magnetic nanostructures. Phys. Rev. Lett. 93, 096806 (2004).

4. Tatara, G., Kohno, H., Shibata, J., Lemaho, Y. \& Lee, K. J. Spin torque and force due to current for general spin textures. J. Phys. Soc. Jpn 76, 054707 (2007).

5. Neubauer, A. et al. Topological Hall effect in the A phase of MnSi. Phys. Rev. Lett. 102, 186602 (2009).

6. Lee, M., Kang, W., Onose, Y., Tokura, Y. \& Ong, N. P. Unusual Hall effect anomaly in MnSi under pressure. Phys. Rev. Lett. 102, 186601 (2009).

7. Fu, L., Kane, C. L. \& Mele, E. J. Topological insulators in three dimensions. Phys. Rev. Lett. 98, 106803 (2007).

8. Zhang, H. et al. Topological insulators in $\mathrm{Bi}_{2} \mathrm{Se}_{3}, \mathrm{Bi}_{2} \mathrm{Te}_{3}$ and $\mathrm{Sb}_{2} \mathrm{Te}_{3}$ with a single Dirac cone on the surface. Nature Phys. 5, 438-442 (2009).

9. Yu, R. et al. Quantized anomalous Hall effect in magnetic topological insulators. Science 329, 61-64 (2010).

10. Nomura, K. \& Nagaosa, N. Surface-quantized anomalous Hall current and the magnetoelectric effect in magnetically disordered topological insulators. Phys. Rev. Lett. 106, 166802 (2011).

11. Chang, C.-Z. et al. Experimental observation of the quantum anomalous Hall effect in a magnetic topological insulator. Science 340, 167-170 (2013).

12. Checkelsky, J. G. et al. Trajectory of the anomalous Hall effect towards the quantized state in a ferromagnetic topological insulator. Nature Phys. 10, 731-736 (2014).

13. Kou, X. et al. Scale-invariant quantum anomalous Hall effect in magnetic topological insulators beyond the two-dimensional limit. Phys. Rev. Lett. 113, 137201 (2014).

14. Chang, C.-Z. et al. High-precision realization of robust quantum anomalous Hall state in a hard ferromagnetic topological insulator. Nature Mater. 14, 473-477 (2015).

15. Mühlbauer, S. et al. Skyrmion lattice in a chiral magnet. Science 323, 915-919 (2009).

16. Yu, X. Z. et al. Real space observation of a two-dimensional skyrmion crystal. Nature 465, 901-904 (2010).

17. Nagaosa, N. \& Tokura, Y. Topological properties and dynamics of magnetic skyrmions. Nature Nanotech. 8, 899-911 (2013).

18. Zhang, J. et al. Band structure engineering in $\left(\mathrm{Bi}_{1-x} \mathrm{Sb}_{x}\right)_{2} \mathrm{Te}_{3}$ ternary topological insulators. Nature Commun. 2, 574 (2011).

19. Kou, X. et al. Manipulating surface-related ferromagnetism in modulation-doped topological insulators. Nano Lett. 13, 4587-4593 (2013).

20. Yoshimi, R. et al. Quantum Hall states stabilized in semi-magnetic bilayers of topological insulators. Nature Commun. 6, 8530 (2015).

21. Yoshimi, R. et al. Dirac electron states formed at the heterointerface between a topological insulator and a conventional semiconductor. Nature Mater. 13, 253-257 (2014).

22. Yoshimi, R. et al. Quantum Hall effect on top and bottom surface states of topological insulator $\left(\mathrm{Bi}_{1-x} \mathrm{Sb}_{x}\right)_{2} \mathrm{Te}_{3}$ films. Nature Commun. 6, 6627 (2015).

23. Girvin, S. M. in The Quantum Hall Effect: Novel Excitations and Broken Symmetries, in Topological Aspects of Low Dimensional Systems (eds Comtet, A., Jolicoeur, T., Ouvry, S. \& David, F.) 55-168 (Springer, 2000).

24. Fert, A., Cros, V. \& Sampaio, J. Skyrmions on the track. Nature Nanotech. 8, 152-156 (2013).

25. Heinze, S. et al. Spontaneous atomic-scale magnetic skyrmion lattice in two dimensions. Nature Phys. 7, 713-718 (2011).

26. Jiang, W. et al. Blowing magnetic skyrmion bubbles. Science 349, 283-286 (2015).

27. Moreau-Luchaire, C. et al. Additive interfacial chiral interaction in multilayers for stabilization of small individual skyrmions at room temperature. Nature Nanotech. http://dx.doi.org/10.1038/nnano.2015.313 (2016).

28. King, P. D. C. et al. Rashba spin-splitting control at the surface of the topological insulator $\mathrm{Bi}_{2} \mathrm{Se}_{3}$. Phys. Rev. Lett. 107, 186405 (2011).

29. Taguchi, Y., Oohara, Y., Yoshizawa, H., Nagaosa, N. \& Tokura, Y. Spin chirality, Berry phase, and anomalous Hall effect in a frustrated ferromagnet. Science 291, 2573-2576 (2001).

30. Ueda, K. et al. Topological Hall effect in pyrochlore lattice with varying density of spin chirality. Phys. Rev. Lett. 108, 156601 (2012).

31. Wakatsuki, R., Ezawa, M. \& Nagaosa, N. Domain wall of a ferromagnet on a three-dimensional topological insulator. Sci. Rep. 5, 13638 (2015). 


\section{Acknowledgements}

We thank J. Matsuno and T. Yokouchi for fruitful discussions and experimental support. This research was supported by the Japan Society for the Promotion of Science through the Funding Program for World-Leading Innovative R\&D on Science and Technology (FIRST Program) on 'Quantum Science on Strong Correlation' initiated by the Council for Science and Technology Policy and by JSPS Grant-in-Aid for Scientific Research(S) No. 24224009 and No. 24226002 from MEXT, Japan

\section{Author contributions}

K.Y. conducted the thin film growth and device fabrication with help from R.Y. K.Y. carried out transport/magnetization measurements. T.M. and R.W. carried out numerical calculations of the Hall conductivity and the skyrmion stability, respectively. K.Y., T.M., R.W., A.T., M.K., N.N. and Y.T. wrote the manuscript with contributions from all the authors. M.K., N.N. and Y.T. conceived and guided the project.

\section{Additional information}

Supplementary information is available in the online version of the paper. Reprints and permissions information is available online at www.nature.com/reprints.

Correspondence and requests for materials should be addressed to K.Y.

\section{Competing financial interests}

The authors declare no competing financial interests. 


\section{Methods}

MBE thin film growth. Thin films were grown by molecular-beam epitaxy (MBE) on insulating InP (111) substrates. The growth temperatures for BST and CBST are $220^{\circ} \mathrm{C}$ and $200^{\circ} \mathrm{C}$, respectively. The $\mathrm{Cr}(x \sim 0.2)$ content is estimated from the beam equivalent pressure (BEP) ratio through $\mathrm{Cr} /(\mathrm{Bi}+\mathrm{Sb})$. The Te flux $\left(\mathrm{BEP}=1.0 \times 10^{-4} \mathrm{~Pa}\right)$ was over-supplied while keeping the $\mathrm{Te} /(\mathrm{Bi}+\mathrm{Sb})$ ratio close to 20 to suppress Te vacancies. Under this condition, the growth rate is about $0.2 \mathrm{~nm} \mathrm{~min}{ }^{-1}$. Before the growth of the first layer, a monolayer $\mathrm{Sb}_{2} \mathrm{Te}_{3}$ buffer layer was grown to give a better morphology and epitaxy. After epitaxial growth of the BST layer, annealing under exposure to the Te flux was performed in situ at $380^{\circ} \mathrm{C}$ to get a smoother surface. The same procedure was employed for the following CBST layer. To suppress degradation of the film, an $\mathrm{AlO}_{x}$ capping layer with a thickness of approximately $3 \mathrm{~nm}$ was deposited at room temperature by an atomic layer deposition system immediately after removing the samples from the MBE vacuum chamber. Please note that $\mathrm{Cr}$ interdiffusion in the heterostructure is negligible, as confirmed by energy-dispersive X-ray spectroscopy imaging (see Supplementary Information). A list of sample structures, such as film thickness, and Bare or FET (including applied gate voltages), is summarized in Supplementary Note 1 and Supplementary Table 1.

FET device fabrication. The Hall-bar device pattern was defined by a photolithographic technique and Ar ion-milling processes. After defining the Hall-bar structure, $\mathrm{AlO}_{x}$ with a thickness of approximately $20 \mathrm{~nm}$ was deposited to serve as a gate capacitor. For Ohmic-contact electrodes and a top gate electrode, 5-nm Ti/45-nm Au were deposited by means of an electron-beam evaporator.

Transport and magnetization measurements. Transport measurements for the bare films were conducted using the d.c. transport option of a physical property measurement system (PPMS, Quantum Design). FET devices were measured in the PPMS with a standard lock-in technique at low frequency $(\sim 7 \mathrm{~Hz})$ and with a low excitation current $(10 \mathrm{nA})$ to suppress heating effects. Low-temperature $(<2 \mathrm{~K})$ measurements were performed using the ${ }^{3} \mathrm{He}$ option of the PPMS. Magnetization measurements for the bare film of 2-nm CBST/5-nm BST were conducted using a magnetic property measurement system (MPMS, Quantum Design). All the transport and magnetization data were anti-symmetrized as a function of the magnetic field.

Calculation of band structures and anomalous Hall conductivity. Band structures and anomalous Hall conductivity were calculated using the low-energy effective Hamiltonian of the TI heterostructures given by

$$
\begin{aligned}
H= & v_{\mathrm{F}} \sum_{i=x, y, z} \hbar k_{i} \sigma_{i} \tau_{x}+\left[m+D_{1}\left(k_{x}^{2}+k_{y}^{2}\right)\right] \tau_{z} \\
& +D_{2}\left(k_{x}^{2}+k_{y}^{2}\right)+U(z)+\left(J_{0}(z)-J_{3}(z) \tau_{z}\right) \sigma_{z}
\end{aligned}
$$

Here, $\sigma_{i}$ and $\tau_{i}$ are the Pauli matrices for the spin and orbital degrees of freedom. $\tau_{z}=+1$ for $\mathrm{Bi} / \mathrm{Sb} p$-orbitals and $\tau_{z}=-1$ for Te $p$-orbitals. The $x$ and $y$ directions are parallel to the TI thin films and periodic, whereas the $z$ direction is perpendicular to the TI thin films and not periodic. Therefore, $k_{z}$ should be regarded as the operator $-i \partial_{z}$. We adopted the following parameters in numerical calculations: $v_{\mathrm{F}}=5.0 \times 10^{5} \mathrm{~m} \mathrm{~s}^{-1}, m=-300 \mathrm{meV}, D_{1}=60 \mathrm{eV} \AA^{2}, D_{2}=20 \mathrm{eV} \AA^{2}$ inside the TI thin films $s^{8}$. The asymmetry between the conduction and valence bands is captured by the $D_{2}$ and $J_{3}$ term. We introduced the potential asymmetry between BST and CBST by setting $U(z)=0 \mathrm{meV}$ for BST and $U(z)=-40 \mathrm{meV}$ for CBST, and the exchange couplings in magnetic TI by setting $J_{0}=J_{3}=0 \mathrm{meV}$ for BST and $J_{0}=5 \mathrm{meV}$ and $J_{3}=1 \mathrm{meV}$ for CBST. Here, the parameters $J_{0}$ and $J_{3}$ are symmetric and asymmetric parts of the exchange couplings for the two orbitals ${ }^{31}$. The exchange coupling for Te $p$-orbitals should be slightly larger than that for $\mathrm{Bi} / \mathrm{Sb}$ $p$-orbitals because Te atoms locate nearer to doped Cr ions. (This asymmetry is captured by setting $J_{3}=0.2 J_{0}$.) The anomalous Hall conductivity is obtained by integrating the Berry curvature $B_{k}=\nabla \times A_{k}$ over the momentum space, where $A_{k}$ is the Berry connection $A_{k}=-i\left\langle\psi_{k}\left|\partial_{k}\right| \psi_{k}\right\rangle$ with Bloch wavefunctions $\psi_{k}$. As seen in Fig. 3e, the valence top bands show Rashba splitting due to the broken inversion symmetry in the TI heterostructure. This Rashba splitting results in the concentration of the Berry curvature at the valence top bands and a large contribution to the anomalous Hall conductivity there.

Calculation of skyrmion stability. The stability of a skyrmion was calculated by the three-dimensional tight-binding model for TI, corresponding to the effective Hamiltonian in the continuum approximation given in equation (1),

$$
\begin{aligned}
H= & t \sum_{i=x, y, z} \sin k_{i} \sigma_{i} \tau_{x}+\left[m+2 D_{1} \sum_{i=x, y, z}\left(1-\cos k_{i}\right)\right] \tau_{z} \\
& +2 D_{2} \sum_{i=x, y, z}\left(1-\cos k_{i}\right)-\left(J_{0}(z)-J_{3}(z) \tau_{z}\right) \mathbf{n} \cdot \sigma
\end{aligned}
$$

where $\sigma_{i}$ and $\tau_{i}$ are the Pauli matrices for the spin and the orbital degrees of freedom. The system size is $L_{x}=L_{y}=31$ sites, $L_{z}=8$ layers, and the periodic boundary condition for the $x$ and $y$ directions is imposed. In this model, the $D_{2}$ and $J_{3}$ terms break the particle-hole symmetry ${ }^{31}$ as mentioned above, and they lead to asymmetry between the electron-doped and hole-doped cases. We have set $D_{1}=1, D_{2}=0.2, t=1, m=-1$, which realizes the strong TI phase. The exchange couplings in magnetic TI are set as $J_{0}=J_{3}=0$ for BST and $J_{0}=0.1$ and $J_{3}=0.02$ for CBST. This choice of the parameters is determined to reduce the finite-size effect, and hence the numerical results are not regarded as quantitatively corresponding to the real system, but rather as qualitative information.

The skyrmion configuration $\mathbf{n}(\mathbf{r})$ is set as

$$
\begin{gathered}
n_{x}=\sin \left[\pi\left(1-\frac{r}{R}\right)\right] \cos [\theta+\phi] \\
n_{y}=\sin \left[\pi\left(1-\frac{r}{R}\right)\right] \sin [\theta+\phi] \\
n_{z}=\cos \left[\pi\left(1-\frac{r}{R}\right)\right]
\end{gathered}
$$

for $r<R$, and $n_{z}=1$ for the outer region $r<R$. The skyrmion radius $R$ is set as the variational parameter and $\phi$ determines the magnetic helicity or the skyrmion type-that is, the Bloch type for $\phi=(\pi / 2)$, the Néell type for $\phi=0$ and the Néel2 type for $\phi=\pi$. We note that $R=0$ corresponds to the ferromagnetic state. One can interpret the obtained results presented in the main text from the facts that valence bands are more strongly coupled to the magnetic ions as a result of the $J_{3}$ term, and the DM interaction is induced from the spin-momentum-locking in the surface state of TI. 